\title{
Non-equilibrium temperatures and heat transport in nanosystems with defects, described by a tensorial internal variable
}

\author{
Liliana Restuccia ${ }^{1 *}$ \\ ${ }^{1}$ University of Messina, Department of Mathematical and Computer Sciences, \\ Physical Sciences, Earth Sciences, Messina, Italy \\ *Email address for correspondence: lrestuccia@unime.it \\ Communicated by Vito Antonio Cimmelli and David Jou \\ Received on January 14, 2015. Accepted on June 14, 2015.
}

\begin{abstract}
The paper deals with the meaning of non-equilibrium temperatures in nanosystems with an internal variable, describing defects inside them, and implications on heat transport. In equilibrium all definitions of temperature lead to the same value, but in nonequilibrium steady states they lead to different values, giving information on different degrees of freedom. We discuss the caloric and entropic non-equilibrium temperatures and the relations among them, in defective nanosystems (crystals with dislocations or porous channels, carbon nanotubes in a solid matrix and so on), crossed by an external energy flux. Here, we present a model for nanocrystals with dislocation defects submitted to an external energy flux. The dislocations may have a strong influence on the effective thermal conductivity, and their own dynamics may be coupled in relevant way to the heat flux dynamics. In the linear case the constitutive relations, the rate equations for the internal variable and the heat flux are worked out and a generalized telegraphic heat equation is derived in the anisotropic and isotropic case, describing the thermal disturbances with finite velocity.

Keywords: Non-equilibrium Thermodynamics, internal variables, defects of dislocation, nanosystems

AMS subject classification: $80 \mathrm{~A} 20,74 \mathrm{~A} 20$
\end{abstract}

\section{Introduction.}

The meaning of temperature in nanosystems is an open problem because of two main reasons: their small size, which makes that the system exhibits large fluctuations in contrast to macroscopic systems, and the strong gradients caused by relatively small temperature differences, which make that the system may be far from local equilibrium. This makes that the meaning 


\section{Restuccia}

of temperature, as well as its role on heat transport, is a relevant topic in nanosystems. On the other side, internal variables may also play an important role in the internal energy and the heat conductivity of the system, which may depend on the internal variables. For instance, the thermal conductivity may depend on the defects or the pores of the material. This dependence influences the heat flux across the system but, reciprocally, the heat flux may modify, in some occasions, the dynamics of the defects or the pores, in such a way that the problem of heat transport may be a strongly coupled in such systems. This may be especially important when the defects or the pores are very small, of the order of the mean free path of the heat carriers in the system (as, for instance, the phonon mean free path in crystals). A relatively high temperature gradient could produce, for instance, a migration of defects inside the system. In this paper, we propose an analysis of heat transport in a system with dislocations, which will act as defects on the thermal conductivity. We analyze their possible role on the internal energy and the entropy and, thus, on the temperature based on internal energy (caloric temperature) and on the entropy (entropic temperature). For instance, if the heat flux is very high, the effective temperature of the internal variables could be different than that of the global system, thus modifying the usual relations between heat transport and temperature gradients.

In Section 2 of this paper we introduce the equilibrium temperature versus non-equilibrium temperatures (see [1], [2], [3], [4], [5], [6], [7] and [8] for applications on this subject).

In Sections 3 and 4, in the framework of extended irreversible thermodynamics with internal variables (see [7], [8], [9] and [10]), a model, developed by the author, is presented for defective nanosystems (crystals with dislocations or porous channels, carbon nanotubes in a solid matrix and so on) [11], [12], [13] and [14], submitted to an external energy flux, where the internal structure is described by a dislocation tensor $\grave{a}$ la Maruszewski (see [15] and [16]) and its gradient.

In Section 5 we discuss the caloric and entropic non-equilibrium temperatures, and the relations among them, in these systems.

Finally in Section 6, as new results, in the linear case, the constitutive relations, the rate equations for the internal variable and the heat flux are worked out, and a generalized telegraphic heat equation (in presence of a dislocation field), with finite velocity for the thermal disturbances, is derived in the anisotropic and isotropic case (see [17], [18] and [19] for studies on the heat conduction in nanosystems in extended thermodynamics). This study has a technological interest in very miniaturized systems (nanotechnology), in high-frequency processes or in the production of new materials with 
Non-equilibrium temperatures and heat transport in nanosystems

sophisticated microstructures and particular thermal properties.

\section{Modeling defective nanosystems in the framework of extended thermodynamics}

In this section we discuss about the limits of validity to model defective nanosystems in the framework of extended thermodynamics (see [9] and [10]).

In fact, in classical irreversible thermodynamics it is assumed the local equilibrium hypothesis, implying that all the variables defined in equilibrium thermodynamics remain significant, and the system under consideration can be mentally split into thermodynamic cells sufficiently large to allow them to be treated as macroscopic thermodynamic subsystems, but sufficiently small so that each cell is in thermodynamic equilibrium. In each cell all the variables (like temperature and entropy) remain uniform but they take different values from cell to cell, they are also allowed to change in the course of time in such a way that they depend continuously on the space and time coordinates. The relationships in equilibrium thermodynamics among state variables remain valid outside equilibrium provided that they are stated locally at each instant of time. The entropy outside equilibrium will depend on the same state variables as at equilibrium. Then, thermodynamic potentials and, consequently, the equations of state of the system, keep their usual equilibrium form but at a local level, namely, for sufficiently small volume elements. The size $d$ of these volumes should be bigger than the average distance, traveled for instance by the heat carriers between two successive collisions, defined mean free path $l(d>l)$. Furthermore, from the point of view of the temporal behavior, because these small volume elements have a characteristic time (called relaxation time), let us say $\tau$, to reach internal equilibrium, it is supposed that inside them the rate of variation of the studied properties is slower than their relaxation time.

In extended thermodynamics we study the behavior of nanosystems [10], where we have situations of high-frequency thermal waves. Inside the volume elements the rate of variation of the physical properties is faster than the time scale characterizing the relaxation of the fluxes towards their respective local-equilibrium value and the perturbations are so fast that their frequency becomes of the order of the reciprocal of the internal relaxation time, given, for instance, by the collision time of heat carriers. Furthermore, the volume element size of these systems along some direction is so small that it becomes comparable to (or smaller than) the mean-free path of the heat carriers $(d \leq l)$. Thus, both situations have a clear physical meaning and, nowadays, a technological interest. Then, in extended thermodynamics it is essential to incorporates the fluxes among the state variables of the system and to establish the spatial rate of variation of the intensive thermodynamic variables in a given non-equilibrium steady state. Such states are the natural generalization of equilibrium states: in them the values of the variables do not depend on time but, in contrast to equilibrium states, a continuous flux of energy - or matter, or momentum, or charge - must be supplied or extracted from the system to keep the inhomogeneous distribution of the values independent on time.

Also, it is interesting, when we consider a system submitted to a heat flow, to define the characteristic residence time $t_{\text {res }}$ of the energy in a volume element 


\section{Restuccia}

of the system (see [10]), i.e. the average time during which the energy supplied to this volume element of the system will stay in it before leaving it, that is of the order of $U / A q, U$ being the internal energy and $A q$ the total heat flux supplied to this material volume element (and leaving the system) per unit time, with $A$ the transverse area through which the heat flux is arriving. Then, in the fast-varying states but also in steady states, if the heat flux is high enough, the energy residence time will be of the order, or even shorter, than the relaxation time $\tau, t_{r e s} \leq \tau$, in such a way that the energy entering into the material volume element will not have time enough to distribute itself among the several degrees of freedom of the element before leaving it. In local equilibrium hypothesis $t_{r e s}$ is longer than $\tau$, $t_{\text {res }}>\tau$.

Thus, in local equilibrium hypothesis for a rigid body, we can use Fourier law for the heat flow propagation, that leads to a parabolic equation describing a diffusive propagation of thermal disturbances with infinite velocity (see [20] and [21]). In extended irreversible thermodynamics Vernotte-Cattaneo equation removes this paradox introducing the relaxation time of the heat flux, leading to the telegraphic heat conduction equation, that allows a thermal propagation with finite speed.

\section{Equilibrium temperatures versus non-equilibrium tempera- tures}

There are different definitions of temperature: thermometric, caloric, entropic, kinetic, vibrational, configurational, fluctuational, and so on (see [1], [2], [3], [4], [5], [6], [7] and [8] ). In this section we introduce the definitions of caloric, entropic and thermometric temperature and we compare them in equilibrium thermodynamics and in non-equilibrium thermodynamics in steady states.

In fact, equilibrium thermodynamics is restricted to equilibrium states. Nonequilibrium steady states are different than equilibrium states because in them the material system is crossed by fluxes of energy, matter, electric current and so on. Thus, it is interesting to investigate the influence of such fluxes on the thermodynamics of the system. The presence of fluxes is related to inhomogeneities in the system: presence of a gradient of temperature, concentration, electrical potential or barycentric velocity.

The equilibrium and non-equilibrium entropic temperatures $\theta_{e q}$ and $\theta_{n e q}$, appearing in Gibbs equation, are defined by the second law of thermodynamics as follows

$$
\theta_{e q}^{-1} \equiv\left(\frac{\partial S_{e q}}{\partial u}\right)_{\tilde{\mathbf{q}}=0} ; \quad \theta_{n e q}^{-1} \equiv\left(\frac{\partial S}{\partial u}\right)_{\tilde{\mathbf{q}} \neq 0},
$$

where $S_{e q}$ is the entropy density per unit mass in the local equilibrium hypothesis, introduced in equilibrium thermodynamics, where each thermodynamic state is an equilibrium state, $S$ is the non-equilibrium entropy density per unit mass, constitutive function of the independent variables belonging to a thermodynamic state space, characterizing the version of non-equilibrium thermodynamic theory, used for the description of the system under consideration (in our case extended irreversible thermodynamics), and $\tilde{\mathbf{q}}$ is a given external energy flux, to which the 


\section{Non-equilibrium temperatures and heat transport in nanosystems}

system is submitted. In an equilibrium thermodynamic state the internal production of entropy vanishes, all the fluxes, the sources of energy, matter, force, and so on, are null, the barycentric velocity of the volume element of a system is constant or vanishing, the spatial gradient and the time derivative of fields present inside the body are null.

The equilibrium and the non-equilibrium caloric temperatures $T_{e q}$ and $T_{n e q}$ are based on the first law of thermodynamics and they are called caloric because they use the so-called caloric equation of state relating internal energy and temperature, obtained from the internal energy of the different degrees of freedom.

Out of equilibrium, energy equipartition is not valid in general; this is a consequence of the fact that the distribution function has no longer the canonical distribution form, namely $e x p^{-\beta H}$, with $H$ the Hamiltonian operator and $\beta$ the reciprocal of absolute temperature, $\beta=1 / k_{B} T$, in classical systems. Thus, the average energy of the several different degrees of freedom is different from that they would have in equilibrium, for a given internal energy of the system. This means, for instance, that if in an ideal gas $k_{B} T=(2 / 3) U=(1 / 3) N m v^{2}$, with $N$ the number of particles, $m$ the mass of a particle, and $v$ the speed of a particle, the temperatures defined from the average kinetic energy along the different axes $\mathrm{x}, \mathrm{y}$ and $\mathrm{z}$ will not be necessarily equal. It follows from here that the empirical temperature will depend on the spatial direction of the thermometers (for instance, from their relative direction with respect to a heat flux in the system). It also follows that for a given internal energy, the entropy of the system is expected to be lower if the temperature along each direction is different that if the temperature was the same; as a consequence, the relation between entropic temperature and caloric temperature will be different from that in equilibrium.

The equilibrium empirical (thermometric) temperature $\theta_{\text {emp,eq }}$ is defined by the zeroth law, which states the transitive character of thermal equilibrium. This principle is not generally valid in non-equilibrium steady states. Indeed, as it has been said, different thermometers sensitive to different degrees of freedom are expected to indicate different temperatures. Therefore, the only possible generalization of zeroth principle (if any generalization is indeed possible) would be one restricted to every different degree of freedom, namely, postulating that if systems $\mathrm{A}$ and $\mathrm{B}$ are in mutual thermal equilibrium (no heat flows between them) being in thermal contact through a given degree of freedom, and systems B and C are in mutual thermal equilibrium being in thermal contact through the same degree of freedom, then systems $\mathrm{A}$ and $\mathrm{C}$ will be in mutual thermal equilibrium if they are in thermal contact through the same degree of freedom as in the above situations. However, if $\mathrm{A}$ and $\mathrm{B}$ are in mutual thermal equilibrium through one degree of freedom, and $\mathrm{B}$ and $\mathrm{C}$ are in mutual thermal equilibrium through a different degree of freedom, $\mathrm{A}$ and $\mathrm{C}$ will not be necessarily in mutual thermal equilibrium. As we have commented, it is not logically necessary that any generalization of the zeroth principle is valid out of equilibrium, neither in the case of restricting it to a given degree of freedom. However, if this were indeed impossible to generalize the zeroth principle to non-equilibrium steady states in the restrictive conditions mentioned here, the use of any kind of thermometer would be useless. It seems however that thermometers are still being used in non-equilibrium steady states, although with 


\section{Restuccia}

some restrictive precautions. This indicates that postulating the validity of the mentioned restriction version of the zeroth principle, though merely tentative, is not completely unreasonable.

Then, in equilibrium thermodynamics different definitions of equilibrium temperature lead to the same value $\theta_{e m p, e q}=\theta_{e q}=T_{e q}$.

Out of equilibrium, in presence of an external energy flux, these nonequilibrium temperatures are different from each other $\theta_{\text {emp }, \text { neq }} \neq \theta_{\text {neq }} \neq T_{\text {neq }}$ (see [7]).

In this contribution, in the framework of extended irreversible thermodynamics, we investigate the caloric and entropic non-equilibrium temperatures and the relations among them in nanosystems with internal structure, described by a tensorial internal variable (crystals with dislocations or porous channels, carbon nanotubes in a solid matrix and so on), when they are crossed by an external energy flux.

\section{Dislocation dynamics, energy flux and non-equilibrium ther- modynamics}

In this section we present a model, developed in the framework of extended thermodynamics by the author in [11] (see also [12]), to describe a nanocrystal with dislocations, and we suppose that it is submitted to an external energy flux $\tilde{\mathbf{q}}$. The following fields interact with each other inside the system: the thermal field, described by the non-equilibrium absolute temperature $\theta_{n e q}$, its gradient $\theta_{n e q, i}$ and the heat flux $q_{i}$; the elastic field described by the stress tensor $\sigma_{i j}$ and the small strain tensor $\varepsilon_{i j}$, defined as $\varepsilon_{i j}=\frac{1}{2}\left(u_{i, j}+u_{j, i}\right)$, with $u_{i}$ the components of the displacement vector; the dislocation field modeled by a dislocation tensor $a_{i j}$ and its gradient $a_{i j, k}$, describing the non-local effects of these dislocation lines. In [11] also the dislocation flux, describing the selfpropagation of dislocations because of changed surrounding conditions that are favorable, was taken into consideration also as internal variable, but here we neglect its influence.

The tensor $a_{i j}$, introduced by Maruszewski in [15], models the local structure of dislocations lines, which form a network of very thin lines, having the same geometrical structure of porous channels, disturbing the otherwise perfect periodicity of the crystal lattice [16] (see Fig.1). Their existence should not be omitted in the analysis of kinetic processes as diffusion of mass or charges, transport of heat and so on. In Fig. 1 a representative elementary sphere volume $\Omega$ provides a statistical representation of the properties of a structure of capillary channels, $\Omega=\Omega^{s}+\Omega^{c h}$, with $\Omega^{s}$ the solid-space and $\Omega^{c h}$ the channel-space. Furthermore, we indicate by $\Gamma$ the sphere central section area, with $\Gamma=\Gamma^{s}+\Gamma^{c h}$, where $\Gamma^{c h}$ is the channel-area of $\Gamma$ and $\Gamma^{s}$ the solid-area. The orientation of $\Gamma$ in $\Omega$ is given by the normal vector $\boldsymbol{\mu}$. The analysis is restricted to media which are homogeneous with respect to the effective channel-volume $f_{v}$, with $f_{v}=\frac{\Omega^{c h}}{\Omega}$, constant in the medium. To avoid confusion, all microscopic quantities are described with respect to the $\xi_{i}$ coordinate system, while macroscopic quantities are described with respect to the $x_{i}$ coordinate system. Then, let $\boldsymbol{\alpha}(\boldsymbol{\xi})$ be any scalar, spatial vector or second order tensor quantity, describing a microscopic property of the flux of 


\section{Non-equilibrium temperatures and heat transport in nanosystems}

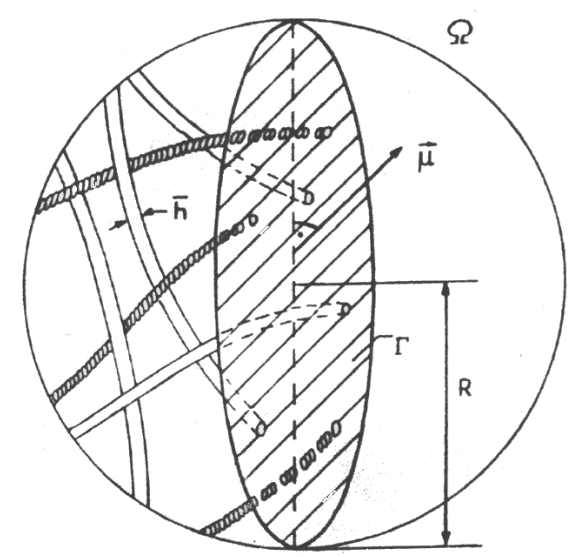

Figure 1. Characteristics of a local channel structure with $(\bar{h} \ll R), h$ diameter of the core and $R$ size of the volume element (after [15])

some physical field, flowing through the channel-space $\Omega^{c h}$, with respect to the $\xi_{i}$ coordinates. We assume that such quantity is zero in the solid-space $\Omega^{s}$ and on the solid-area $\Gamma^{s}$. In such a medium Maruszewski defines the so called dislocation tensor, $\mathcal{R}_{i j}$, as follows [15] (see also [16])

$$
\bar{\alpha}(\mathbf{x})_{i}=\mathcal{R}_{i j}(\mathbf{x}, \boldsymbol{\mu}) \stackrel{*}{\alpha},(\mathbf{x}, \boldsymbol{\mu}) .
$$

Eq. (2) gives a linear mapping between the bulk-volume average quantity $\overline{\boldsymbol{\alpha}}(\mathbf{x})$ and the channel-area average of the same quantity $\stackrel{*}{\boldsymbol{\alpha}}(\mathbf{x}, \boldsymbol{\mu})$, given by

$$
\overline{\boldsymbol{\alpha}}(\mathbf{x})=\frac{1}{\Omega} \int_{\Omega^{c h}} \boldsymbol{\alpha}(\boldsymbol{\xi}) d \Omega, \quad \stackrel{*}{\boldsymbol{\alpha}}(\mathbf{x}, \boldsymbol{\mu})=\frac{1}{\Gamma^{c h}} \int_{\Gamma} \boldsymbol{\alpha}(\boldsymbol{\xi}) d \Gamma,
$$

where the quantities $\overline{\boldsymbol{\alpha}}(\mathbf{x})$ and $\stackrel{*}{\boldsymbol{\alpha}}(\mathbf{x})$ describe at macroscopic level the same property of the flux of the physical field under consideration. In [16] Kubik gives for a structure with thin channels an interpretation of $\mathcal{R}_{i j}$, considering the flux of a quantity $\overline{\boldsymbol{\alpha}}(\mathbf{x})$ on a bulk-volume as a superposition of three one-dimensional fluxes (along three mutually perpendicular channels) having average values $\stackrel{*}{\alpha}_{i}(\mathbf{x}, \boldsymbol{\mu})$ on the orthogonal section areas of these channels. In [15] a new tensor, that refers $\mathcal{R}_{i j}$ to the surface $\Gamma$, is defined in the following way

$$
\mathcal{R}_{i j}(\mathbf{x}, \boldsymbol{\mu})=\Gamma a_{i j}(\mathbf{x}, \boldsymbol{\mu}) .
$$

$a_{i j}$ is called dislocation core tensor and its unit is $m^{-2}$. The components of $a_{i j}$ form a kind of continuous representation of the number of dislocations which cross the surface $\Gamma$. Investigations show that during many physical processes occurring in a defective crystal the temporal evolution of the dislocation field is of great importance $\left(a_{i j}\right.$ is also dependent on time). But, whereas the classical variables as $\theta$ and $\epsilon_{i j}$ may be measured and controlled at any moment of time, the internal variable $a_{i j}$ may be measured only by suitable methods and cannot be controlled 


\section{Restuccia}

with the same immediateness. They appear in the Gibbs equation like the classical thermodynamical variables.

In our model we use the standard Cartesian tensor notation in a rectangular coordinate system and refer the motion of our material system to a current configuration $\mathcal{K}_{t}$. Then, the state variables are represented by the set

$$
\mathbf{C}=\left\{\varepsilon_{i j}, \theta_{n e q}, \theta_{n e q, i}, a_{i j}, a_{i j, k}, q_{i}\right\},
$$

where the presence of the heat flux permits to take into account thermal relaxation in nanosystems, where the velocity of thermal disturbances is finite.

Thus, all the processes occurring in the considered body are governed by two groups of laws. The first group concerns the following balance equations: the continuity equation

$$
\dot{\rho}+\rho v_{i, i}=0,
$$

where $\rho$ denotes the mass density, the superimposed dot denotes the material time derivative and $v_{i}$ is the velocity field;

the momentum balance:

$$
\rho \dot{v}_{i}-t_{j i, j}-f_{i}=0,
$$

where $f_{i}$ is the body force and $t_{i j}$ is the stress tensor (whose symmetry was demonstrated in [11]);

the internal energy balance:

$$
\rho \dot{U}-\sigma_{i j} \dot{\varepsilon}_{i j}+q_{i, i}+\tilde{q}_{i, i}=0,
$$

where $U$ is the internal energy density and $\tilde{\mathbf{q}}$ is a given additional external heat flux, to which the system is submitted.

The second group of laws deals with the evolution equation of the internal variable (the dislocation core tensor) and the rate equation for the heat flux $\mathbf{q}$, that, in field formulation, we may assume to have the form [22], [23], [24] and [25]

$$
\dot{a}_{i j}+\mathcal{V}_{i j k, k}=A_{i j}(\mathbf{C})+\tilde{A}_{i j}(\tilde{\mathbf{q}})
$$

$$
\dot{q}_{i}=Q_{i}(\mathbf{C})+\tilde{q}_{i}
$$

In $(8)_{1} \mathcal{V}_{i j k}$ is the dislocation flux tensor, $A_{i j}(\mathbf{C})$ is the internal source of dislocations due to the fields present in the system and $\tilde{A}_{i j}(\tilde{\mathbf{q}})$ is the external source connected with a given heat flux $\tilde{\mathbf{q}}$ crossing the system. In $(8)_{2} Q_{i}(\mathbf{C})$ is the heat flux internal source due to the fields inside the body, $\tilde{\mathbf{q}}$ is the external source and the flux tensor of $\mathbf{q}$ is not taken into consideration.

To be sure that the physical processes occurring in the considered body are real, all the admissible solutions of the proposed evolution equations have to satisfy the following entropy inequality

$$
\rho \dot{S}+\nabla \cdot \mathbf{J}^{s}-\frac{\rho r}{\theta} \geq 0,
$$




\section{Non-equilibrium temperatures and heat transport in nanosystems}

with $\mathbf{J}^{s}$ the entropy flux. The set of the constitutive functions are

$$
\mathbf{W}=\left\{\sigma_{i j}, U, A_{i j}, Q_{i}, S, J_{i}^{s}, \pi_{i j}, \Pi_{i}^{q}\right\},
$$

$$
\mathbf{W}=\tilde{\mathbf{W}}(\mathbf{C})
$$

where both $\mathbf{C}$ and $\mathbf{W}$ are evaluated at the same point and time, $\pi_{i j}$ is the thermodynamic potential conjugate to $a_{i j}$ and $\Pi_{i}^{q}$ is the thermodynamic potential conjugate to $q_{i}$. The ensemble of the balance equations, the evolution laws of the internal variable and the heat flux and the constitutive laws, that we will derive as new results in Section 6, describes the evolution of the nanosystem with dislocations under consideration.

In [11] the entropy inequality was analyzed by Liu's theorem [26] and among the other results the laws of state, the affinities, the entropy flux and the form of the free energy, defined by Legendre transformation $F=U-\theta_{n e q} S$, were given in the form:

the laws of state

$$
\rho \frac{\partial F}{\partial \varepsilon_{i j}}=\sigma_{i j} \quad \frac{\partial F}{\partial \theta_{n e q}}=-S, \quad \frac{\partial F}{\partial a_{i j, k}}=0, \quad \frac{\partial F}{\partial \theta_{n e q, i}}=0,
$$

the affinities

$$
\rho \frac{\partial F}{\partial a_{i j}} \equiv \pi_{i j}, \quad \rho \frac{\partial F}{\partial q_{i}} \equiv \Pi_{i}^{q}
$$

and the free energy

$$
F=F\left(\varepsilon_{i j}, \theta_{n e q}, a_{i j}, q_{i}\right)
$$

that is invariant under time reversal.

\section{Non-equilibrium temperatures in a crystal with dislocations}

In this section we discuss the entropic and caloric non-equilibrium temperatures in defective nanosystems in non-equilibrium steady states, when they are crossed by a given energy flux (see [7] and [8]).

The reciprocal non-equilibrium entropic temperature $\theta_{n e q}^{-1}$ can be expanded around the reciprocal equilibrium temperature $\theta_{e q}^{-1}$ obtaining the following result, in first approximation and for given values of the other fields,

$$
\theta_{n e q}^{-1}=\theta_{e q}^{-1}-\theta_{e q}^{-2} \frac{\partial \theta_{e q}}{\partial a_{i j}} \Delta a_{i j}
$$

with

$$
\Delta a_{i j}=a_{i j}(\tilde{\mathbf{q}} \neq 0)-a_{i j}(\tilde{\mathbf{q}}=0)
$$




\section{Restuccia}

Then, in the first approximation, the non-equilibrium temperature $\theta_{n e q}$ will be related to the equilibrium temperature as

$$
\begin{gathered}
\theta_{n e q}=\frac{\theta_{e q}}{1-\theta_{e q}^{-1} \frac{\partial \theta_{e q}}{\partial a_{i j}} \Delta a_{i j}} \approx T_{e q}\left(1+T_{e q}^{-1}\left(\frac{\partial T_{e q}}{\partial a_{i j}}\right) \Delta a_{i j}\right)= \\
T_{e q}+\left(\frac{\partial T_{e q}}{\partial a_{i j}}\right) \Delta a_{i j},
\end{gathered}
$$

where $\theta_{e q}=T_{e q}$ and we have used the approximation $(1-x)^{-1} \approx 1+x$, for $x \ll 1$.

To define the caloric temperature related to the internal variable $a_{i j}$ (see [7] and [8]), first, we consider the caloric equation of state at the equilibrium of the system for given values of $\epsilon_{k l}$ and for vanishing values of the external flux $\tilde{\mathbf{q}}$ :

$$
U_{d i s}=U\left(a_{i j}\left(T_{e q}, \epsilon_{k l}\right), T_{e q}, \epsilon_{k l}\right)
$$

where we have taken into consideration that at equilibrium the internal variable depends on the temperature and the stress tensor. Then, we define the caloric non-equilibrium temperature field $T_{n e q}$ related to $a_{i j}$ in a steady state, assuming that out the equilibrium the internal energy has the same form as in equilibrium in presence of $\tilde{\mathbf{q}}$.

$$
U_{d i s}\left(a_{i j}\left(T_{n e q}, \epsilon_{k l}, \tilde{\mathbf{q}}=0\right), T_{n e q}, \epsilon_{k l}\right) \equiv U_{d i s}\left(a_{i j}\left(T_{e q}, \epsilon_{k l}, \tilde{\mathbf{q}}\right), T_{e q}, \epsilon_{k l}\right) .
$$

Then, in the first approximation the non-equilibrium caloric temperature will be related to equilibrium temperature by

$$
\begin{gathered}
T_{n e q}=T_{e q}+\left(\frac{\partial T_{e q}}{\partial U_{d i s}}\right) \Delta U_{d i s}=T_{e q}+\left(\frac{1}{c_{d i s}}\right) \Delta U_{d i s}= \\
T_{e q}+\left(\frac{\partial T_{e q}}{\partial U_{d i s}}\right)\left(\frac{\partial U_{d i s}}{\partial a_{i j}}\right) \Delta a_{i j},
\end{gathered}
$$

where $c_{d i s}=\frac{\partial U_{d i s}}{\partial T_{e q}}$ is the specific heat associated to the changes of the internal energy of dislocation lines, per unit volume. It is seen that in this order of approximation both non-equilibrium temperatures (entropic and caloric) coincide (see [7] and [8]).

\section{Constitutive theory and heat equation for defective nanosys- tems: linear case}

In this section, as new results, we derive the constitutive relations and the heat equation for a nanosystem with dislocations (see [17], [18] and [19] for studies on the heat conduction in nanosystems in extended thermodynamics). Then, let us consider a reference state of the medium, with an arbitrary (but fixed) uniform 


\section{Non-equilibrium temperatures and heat transport in nanosystems}

temperature $\theta_{0}$. We also require that the reference state (indicated by the symbol "0") is a state of thermodynamic equilibrium. We choose the tensors $\varepsilon_{i j}, a_{i j}$ and the heat flux vector $q_{i}$ so that they vanish in the reference state, and hence

$$
(S)_{0}=S_{0}, \quad\left(\sigma_{i j}\right)_{0}=0, \quad\left(\pi_{i j}\right)_{0}=0, \quad\left(\Pi_{i}^{q}\right)_{0}=0,
$$

$$
\text { for } \quad(\theta)_{0}=\theta_{0}, \quad\left(\varepsilon_{i j}\right)_{0}=0, \quad\left(a_{i j}\right)_{0}=0, \quad\left(q_{i}\right)_{0}=0
$$

Thus, we expand the free energy around this equilibrium state. Denoting the deviations with respect to this state by

(21) $\bar{\theta}_{n e q}=\theta_{n e q}-\theta_{0}, \quad \alpha_{i j}=a_{i j}-\left(a_{i j}\right)_{0}, \quad \bar{\varepsilon}_{i j}=\varepsilon_{i j}-\left(\varepsilon_{i j}\right)_{0}, \quad \bar{q}_{i}=q_{i}-\left(q_{i}\right)_{0}$,

the free energy $F$ takes the form

$F-F_{0}=-S_{0} \theta_{n e q}+\frac{1}{2 \rho} c_{i j l m} \varepsilon_{i j} \varepsilon_{l m}-\frac{\lambda_{i j}^{\theta}}{\rho} \theta_{n e q} \varepsilon_{i j}+\frac{\alpha_{i j l m}^{a \epsilon}}{\rho} \varepsilon_{i j} \alpha_{l m}-\frac{1}{2} \frac{c}{\theta_{0}} \theta_{n e q}^{2}+\frac{\alpha_{i j}^{a \theta}}{\rho} \alpha_{i j} \theta_{n e q}+$

$$
\frac{\alpha_{i j l m}^{a a}}{2 \rho} \alpha_{i j} \alpha_{l m}+\frac{1}{2 \rho} \alpha_{i j}^{q q} q_{i} q_{j},
$$

where the order terms higher than the second one are neglected and the deviation $\bar{\theta}_{n e q}$ continues to be called $\theta_{n e q}$. In this equation the property that $F$ is invariant under time reversal has been used, so that no terms containing $q_{i}$ at first order are present. Furthermore, $c$ denotes the specific heat, $c_{i j l m}$ is the elastic tensor, $\lambda_{i j}^{\theta}$ are the thermoelastic constants and the other quantities express the interactions among the various fields present in the system. Moreover, the constant phenomenological coefficients satisfy the following symmetry relations

$$
\begin{gathered}
c_{i j l m}=c_{l m i j}=c_{j i l m}=c_{i j m l}=c_{j i m l}=c_{m l i j}=c_{m l j i}=c_{l m j i}, \\
\lambda_{i j}^{\theta}=\lambda_{j i}^{\theta}, \quad \alpha_{i j l m}^{a \epsilon}=\alpha_{l m j i}^{a \epsilon}=\alpha_{l m i j}^{a \epsilon}=\alpha_{j i l m}^{a \epsilon}, \quad \alpha_{i j l m}^{a a}=\alpha_{l m i j}^{a a}, \\
\alpha_{i j}^{q q}=\alpha_{j i}^{q q} .
\end{gathered}
$$

Finally, using the laws of state (12) and the definitions of affinities (13), from the approximated expression for the free energy (22), the following expressions are derived

$$
\begin{gathered}
\sigma_{i j}=c_{i j l m} \varepsilon_{l m}-\lambda_{i j}^{\theta} \theta_{n e q}+\alpha_{i j l m}^{a \epsilon} \alpha_{l m}, \\
S=S_{0}+\frac{\lambda_{i j}^{\theta}}{\rho} \varepsilon_{i j}+\frac{c}{\theta_{0}} \theta_{n e q}-\frac{\alpha_{i j}^{a \theta}}{\rho} \alpha_{i j},
\end{gathered}
$$




$$
\pi_{i j}=\alpha_{i j l m}^{a \epsilon} \varepsilon_{l m}+\alpha_{i j}^{a \theta} \theta_{n e q}+\alpha_{i j l m}^{a a} \alpha_{l m},
$$

$$
\Pi_{i}^{q}=\alpha_{i j}^{q q} q_{j} .
$$

Furthermore, from (8) we have the following linearized rate equations for the dislocation tensor and the heat flux, where the constitutive functions $A_{i j}(\mathbf{C})$ and $Q_{i}(\mathbf{C})$ have been constructed as linear objective polonomial expressions in terms of the independent variables (see [27]),

$$
\dot{q}_{i}=\delta_{i j k}^{1} \varepsilon_{j k}+\delta_{i j}^{2} \theta_{n e q, j}+\delta_{i j k}^{3} \alpha_{j k}+\delta_{i j k l}^{4} \alpha_{j k, l}+\delta_{i j}^{5} q_{j}+\tilde{q}_{i},
$$

where $\boldsymbol{\beta}^{i}(i=1,2, \ldots, 5)$ and $\boldsymbol{\delta}^{r}(r=1,2, \ldots, 5)$ are assumed constant phenomenological tensors. The evolution equation for the internal variable (30) could be simplified in the linearized form (see [7], [8])

$$
\frac{d \alpha_{i j}}{d t}-D \nabla^{2} \alpha_{i j}=A_{i j, e q}+\nu \tilde{q}_{i} \tilde{q}_{j},
$$

where it is assumed for the dislocation flux the expression $\mathcal{V}_{i j k}=-D \frac{\partial \alpha_{i j}}{\partial x_{k}}$, with $D$ being a diffusion coefficient for dislocations, and the sources of dislocations reduce to two contributions: an internal source $A_{i j, e q}$, describing the net formation tensor of dislocation lines in an equilibrium state, in the absence of an external energy flux, and a source depending on the external given heat flux $\tilde{q}_{i}$. The form of this last contribution does not pretend to be especially realistic, but only to illustrate that thermal stresses related to $\tilde{q}_{i} \tilde{q}_{j}$ could influence the evolution of dislocation lines. Equation (31) generalizes Vernotte-Cattaneo relation (see [21]), in presence of a small deformation field, a dislocation field and its gradient, that allows finite velocities for thermal disturbances. Denoting by $\tau$ a relaxation time associated to the heat flux, it can take the form

$$
\tau \dot{q}_{i}=-q_{i}-\chi_{i j}^{1} \theta_{n e q, j}+\chi_{i j k}^{2} \varepsilon_{j k}+\chi_{i j k}^{3} \alpha_{j k}+\chi_{i j k l}^{4} \alpha_{j k, l}+\tilde{q}_{i},
$$

where we have indicated with $\chi_{i j}^{1}$ the thermal conductivity tensor and with $\chi_{i j k}^{2}, \chi_{i j k}^{3}, \chi_{i j k l}^{4}$ the tensors connected with the influence of the deformation, dislocation and dislocation gradient fields on the heat flux relaxation, respectively.

Now, we work out the heat conduction equation.

Introducing the free energy $F=U-\theta_{\text {neq }} S$, considering the material derivative of this expression

$$
\rho \theta_{n e q} \dot{S}=\rho \dot{U}-\rho S \dot{\theta}_{n e q}-\rho \dot{F}
$$




\section{Non-equilibrium temperatures and heat transport in nanosystems}

and taking into consideration the balance energy equation (7), we obtain

$$
\rho_{0} \theta_{n e q} \dot{S}=\sigma_{i j} \dot{\varepsilon}_{i j}-q_{i, i}-\tilde{q}_{i, i}-\rho_{0} \dot{\theta}_{n e q} S-\rho_{0} \dot{F}
$$

where $\rho$ is considered practically constant and denoted by $\rho_{0}$.

From (35), calculating the material derivative of the free energy $F=F\left(\varepsilon_{i j}, \theta_{n e q}, a_{i j}, q_{i}\right)$, we have

$$
\begin{aligned}
\rho_{0} \theta_{n e q} \dot{S} & =\sigma_{i j} \dot{\varepsilon}_{i j}-q_{i, i}-\tilde{q}_{i, i}-\rho_{0} \dot{\theta}_{n e q} S-\rho_{0} \frac{\partial F}{\partial \varepsilon_{i j}} \dot{\varepsilon}_{i j}- \\
& -\rho_{0} \frac{\partial F}{\partial \theta} \dot{\theta}_{n e q}-\rho_{0} \frac{\partial F}{\partial a_{i j}} \dot{a}_{i j}-\rho_{0} \frac{\partial F}{\partial q_{i}} \dot{q}_{i} .
\end{aligned}
$$

Finally, using the laws of state and the definitions of the affinities, we derive

$$
\rho \theta_{n e q} \dot{S}=-q_{i, i}-\tilde{q}_{i, i}-\pi_{i j} \dot{a}_{i j}-\Pi_{i}^{q} \dot{q}_{i} .
$$

Neglecting in equations (27) the influence of the strain deformation field and in (33) the influence of the strain deformation field and the gradient of dislocation field, we obtain the following constitutive equation for $S$ and the following rate equation for the heat flux

$$
\begin{gathered}
S=S_{0}+\frac{c}{\theta_{0}} \theta_{n e q}-\frac{\alpha_{i j}^{a \theta}}{\rho} \alpha_{i j}, \\
\tau \dot{q}_{i}=-q_{i}-\chi_{i j}^{1} \theta_{n e q, j}+\chi_{i j k}^{3} \alpha_{j k}+\tilde{q}_{i} .
\end{gathered}
$$

Equation (39) is a generalized Vernotte-Cattaneo equation, in presence of a dislocation field and an external energy flux.

Then, we linearize equation (37) obtaining the heat conduction equation in the form

$$
\rho_{0} \theta_{0} \dot{S}=-q_{i, i}-\tilde{q}_{i, i}, \quad \tau \rho_{0} \theta_{0} \ddot{S}=-\tau\left(\dot{q}_{i, i}+\dot{\tilde{q}}_{i, i}\right)
$$

From $(40)_{2}$, using (38), (39) and $(40)_{1}$, we have

$$
\begin{gathered}
\tau \rho_{0} \theta_{0}\left(\frac{c}{\theta_{0}} \ddot{\theta}_{n e q}-\frac{\alpha_{i j}^{a \theta}}{\rho_{0}} \ddot{\alpha}_{i j}\right)=-\rho_{0} \theta_{0}\left(\frac{c}{\theta_{0}} \dot{\theta}_{n e q}-\frac{\alpha_{i j}^{a \theta}}{\rho_{0}} \dot{\alpha}_{i j}\right)+ \\
+\chi_{i j}^{1} \theta_{n e q, j i}-\chi_{i j k}^{3} \alpha_{j k, i},
\end{gathered}
$$

where we have neglected the influence of the terms containing $\dot{\tilde{q}}_{i, i}$ and $\tilde{q}_{i, i}$. From (41) we obtain

(42) $\tau\left(\frac{c}{\theta_{0}} \ddot{\theta}_{n e q}-\frac{\alpha_{i j}^{a \theta}}{\rho_{0}} \ddot{\alpha}_{i j}\right)+\left(\frac{c}{\theta_{0}} \dot{\theta}_{n e q}-\frac{\alpha_{i j}^{a \theta}}{\rho_{0}} \dot{\alpha}_{i j}\right)-\left(\rho_{0} \theta_{0}\right)^{-1}\left(\chi_{i j}^{1} \theta_{n e q, j i}-\chi_{i j k}^{3} \alpha_{j k, i}\right)=0$ 


\section{Restuccia}

Finally, introducing

$$
\eta_{i j}=\frac{\theta_{0} \alpha_{i j}^{a \theta}}{\rho_{0}}, \quad k_{i j}=\frac{\chi_{i j}^{1}}{\rho_{0}}, \quad \chi_{i j k}=\frac{\chi_{i j k}^{3}}{\rho_{0}},
$$

we obtain a generalized telegraphic heat equation for anisotropic crystals in presence of defects of dislocation

$$
\tau \ddot{\theta}_{n e q}+\dot{\theta}_{n e q}-\frac{\eta_{i j}}{c}\left(\tau \ddot{\alpha}_{i j}+\dot{\alpha}_{i j}\right)=\frac{k_{i j}}{c} \theta_{n e q, j i}-\frac{\chi_{i j k}}{c} \alpha_{j k, i},
$$

where we suppose $\alpha_{i j}$ obeys the rate equation (32).

Now, if we consider the case of isotropic defective solids, having symmetry properties, under orthogonal transformations, which are invariant with respect to all the rotations and inversions of the frame of axes, for the Curie symmetry principle we have:

i) the polar tensor of order two must have the form

$$
\eta_{i j}=\eta \delta_{i j}, \quad k_{i j}=k \delta_{i j},
$$

with $k$ the thermal conductivity,

ii) the polar tensor of order three vanishes

$$
\chi_{i j k}=0 .
$$

The heat equation becomes

$$
\tau \ddot{\theta}_{n e q}+\dot{\theta}_{n e q}-\frac{\eta}{c}\left(\tau \ddot{\alpha}_{i i}+\dot{\alpha}_{i i}\right)=\frac{k}{c} \theta_{n e q, i i},
$$

leading to finite speeds of propagation of thermal disturbances. It is a generalized telegraphic equation for isotropic defective nanosystems. In the case that the solid is perfect (44) reduces to the telegrafic equation

$$
\tau \ddot{\theta}_{n e q}+\dot{\theta}_{n e q}=\frac{k}{c} \theta_{n e q, i i},
$$

whose solution is well known.

\section{Conclusions}

In this paper we have considered a nanosystem with defects submitted to an external energy flux. We have proposed a model in which the defects of dislocations or porous channels are considered as an internal variable with its own dynamics. This allows a richer description of the system than simply assuming the dislocations as a static given parameter. This idea could be also used in simpler systems, as for instance porous systems, where the pores are usually considered as a set of fixed spheres inside the bulk of the body, but without a dynamics. The second point in our model is that we have assumed that an additional heat flux (or an energy flux, in general) may contribute to the dynamics of the defects. This could 


\section{Non-equilibrium temperatures and heat transport in nanosystems}

have interesting consequences on heat transfer. Indeed, the defects obviously have an influence on the thermal conductivity. If the dynamics of the defects depends on the heat flux, the latter one may have a static and a dynamic influence on the thermal conductivity. In particular, if there is some sort of "phonon drag" of defects (i.e. if the heat flux was able to produce a slow drift on the defects) this could open the way to a procedure for improving the so-called "phononic" devices, leading, for instance, to a negative differential heat conductivity, which is necessary to get phononic transistors. This topic is being studied nowadays, and we hope to report on the results in a near future. Another original point of this paper is the detailed consideration of the constitutive equations for internal energy and entropy, and getting, from them, a caloric temperature and an entropic temperature, which are equal at equilibrium but may be different out of equilibrium. The entropic temperature is related to heat transfer, and it may have influence on the effective thermal conductivity, whereas the caloric temperature is related to the energy content and, therefore, it may have an influence on the specific heat. Careful attention to these aspects should be taken in the future.

\section{Acknowledgements.}

The author thanks Prof. David Jou for his discussions, encouragement to write this work, and comments about the non-equilibrium empirical temperature and the non validity of energy equipartition principle out thermodynamic equilibrium. Furthermore, she is grateful to INDAM (GNFM) for a financial support provided to partecipate in FJIM 2014, First Joint International Meeting RSME-SCM-SEMASIMAI-UMI, June 30-July 4, 2014, Bilbao, Spain.

\section{REFERENCES}

1. J. Casas-Vazquez and D. Jou, Temperature in non-equilibrium states: a review of open problems and current proposals, Reports on Progress in Physics, vol. 66bb, p. 1937, 2003.

2. J. G. Powles, G. Rickayzen, and D. M. Heyes, Temperatures: old, new and middle aged., Mol. Phys., vol. 103, pp. 1361-1373, 2005.

3. W. G. Hoover and C. G. Hoover, Non-equilibrium temperature and thermometry in heat-conducting models, Phys. Rev. E, vol. 77, no. 041104, 2008.

4. M. Criado-Sancho, D. Jou, and J. Casas-Vazquez, Non-equilibrium kinetic temperature in flowing gases, Phys. Lett. A, vol. 350, pp. 339-341, 2006.

5. R. S. Johal, Quantum heat engines and non-equilibrium temperature, Phys. Rev. E, vol. 80, no. 041119, 2009.

6. D. Jou, V. A. Cimmelli, and A. Sellitto, Non-equilibrium temperatures and second-sound propagation along nanowires and thin wires, Phys. Lett. A, vol. 373, pp. 4386-4392, 2009.

7. D. Jou and L. Restuccia, Non-equilibrium temperatures in systems with internal variables, in Proceedings of the 12th Joint European Thermodynamics Conference, JETC2013, Brescia, Italy, July, 1-5, 2013 (M. Pilotelli and G. P. Beretta, eds.), University of Brescia, Italy, Cartolibreria SNOOPY s.n.c., 2013. 


\section{Restuccia}

8. D. Jou and L. Restuccia, Temperature, heat transport, and dislocations, to be published on Atti della Accademia Peloritana dei Pericolanti, Proceedings of International Conference Thermal Theories of Continua: Survey and Developments, THERMOCON2016, Messina, April 19-22, 2016, Italy, vol. Supplemento, 2016.

9. D. Jou, J. Casas-Vazquez, and G. Lebon, Extended Irreversible Thermodynamics. Springer, Berlin, fourth edition, 2010.

10. D. Jou and L. Restuccia, Mesoscopic transport equations and contemporary thermodynamics: an introduction, Contemporary Physics, vol. 52, no. 5, pp. 465-474, 2011.

11. L. Restuccia, On a non-conventional thermodynamical model of a crystal with defects of dislocation, to be published.

12. L. Restuccia, Thermomechanics of porous solids filled by fluid flow, in Series on Advances in Mathematics for Applied Sciences, Applied and Industrial Mathematics in Italy III (E. D. Bernardis, R. Spigler, and V. Valente, eds.), vol. 82, pp. 485-495, 2010.

13. F. R. N. Nabarro, Theory of Crystal Dislocations. Clarendon Press, Oxford, 1967.

14. D. Hull, Introduction to Dislocations. Pergamon Press, London, Oxford, 1975.

15. B. Maruszewski, On a dislocation core tensor, Phys. stat. sol.(b), vol. 168, p. 59, 1991.

16. J. Kubik, A macroscopic description of geometrical pore structure of porous solids, Int. J. Engng. Sci., vol. 24, p. 971, 1986.

17. M. Grmela, G. Lebon, and C. Dubois, Multiscale thermodynamics and mechanics of heat, Physical Review E, Statistical, Nonlinear, and Soft Matter Physics, vol. 83, no. 6, p. 061134, 2011.

18. G. Lebon, H. MacHrafi, M. Grmela, and C. Dubois, An extended thermodynamic model of transient heat conduction at sub-continuum scales, Proceedings of the Royal Society A: Mathematical, Physical and Engineering Sciences, vol. 467, pp. 3241-3256, 2011.

19. G. Lebon, Heat conduction at micro and nanoscales: a review through the prism of extended irreversible thermodynamics, J. Non-Equilib Thermodyn., vol. 39, p. 35, 2014.

20. G. Fichera, Is the Fourier theory of heat propagation paradoxical?, Rendiconti del Circolo Matematico di Palermo, vol. II, no. XLI, pp. 5-16, 1992.

21. C. Cattaneo, Sulla conduzione del calore, Atti Sem. Mat. Fis. Univ. Modena, vol. 3, pp. 83-101, 1948.

22. W. Muschik, Aspects of Non-Equilibrium Thermodynamics. World Scientific, Singapore, 1990.

23. W. Muschik, Non-equilibrium thermodynamics with applications to solids, in CISM Courses and Lectures, 336 (W. Muschik, ed.), Springer-Verlag, WienNew York, 1993. 


\section{Non-equilibrium temperatures and heat transport in nanosystems}

24. W. Muschik, C. Papenfuss, and H. Ehrentraut, A sketch of continuum thermodynamics, J. Non-Newtonian Fuid Mech., vol. 96, pp. 255-299, 2001.

25. W. Muschik and L. Restuccia, Terminology and classification of special versions of continuum thermodynamics, Communications to SIMAI Congress, vol. 1, no. 10.1685/CSC06120 ISSN 1827-9015, 2006.

26. I. S. Liu, The method of Lagrange multipliers for exploitation of the entropy principle, Arch. Rat. Mech. Anal., vol. 46, p. 131, 1972.

27. W. Muschik and L. Restuccia, Systematic remarks on objectivity and frameindifference, liquid crystal theory as an example, Archive of Applied Mechanics, no. $10.1007 / \mathrm{s} 00419-007-0193-2$, pp. 1-18, 2008. 